\title{
The celestial reference frame stability and apparent motions of the radio sources
}

\author{
V. E. Zharov ${ }^{1}$, M. V. Sazhin ${ }^{2}$, V. N. Sementsov ${ }^{2}$, K. V. Kuimov ${ }^{2}$, \\ O. S. Sazhina ${ }^{2}$ and N. T. Ashimbaeva ${ }^{2}$ \\ ${ }^{1}$ Physics Departement of Moscow State University, \\ Universitetsky pr. 13, Moscow, Russia \\ email: VladZh2007@yandex.ru \\ ${ }^{2}$ Sternberg State Astronomical Institute of Moscow State University, \\ Universitetsky pr. 13, Moscow, Russia \\ email: valera@sai.msu.ru
}

\begin{abstract}
Time series of the coordinates of the ICRF radio sources were analyzed. It was shown that part of radio sources, including even the so-called "defining" sources, show a the significant apparent motion. Corrections for their a priori coordinates are time functions. The celestial reference frame stability is provided by the no-net-rotation condition applied to the selected subset of sources, which leads in our case to a rotation of the frame axes with time. Parameters of this rotation were calculated for different subsets of sources.

To improve stability of the celestial reference frame new methods of selection of the extragalactic radio sources were suggested. The first one was called "cosmological" and the second one "kinematical". It was shown that a selected subset of the ICRF sources, according to cosmological criteria, determines the most stable coordinate system during next decade.
\end{abstract}

\section{Introduction}

The International Celestial Reference System (ICRS) is based on the positions of 608 selected compact extragalactic radio sources (quasars, active galactic nuclei (AGN), and blazars) (Ma et al., 1998). Stability of the system axes is guaranteed by positions of the "defining" radio sources. One assumes that their coordinates are known as precisely as possible. These sources are unresolved with VLBI baselines comparable to the Earth diameter, and it was assumed that variations of their coordinates are negligible.

To prepare a new catalogue of radio sources (new realization of ICRF-2) one has first to analyze the observation data during 1980-2008 years. The first aim of this analysis was comparison of the time series of the sources' coordinates calculated by different groups which use different software for reduction and analysis of VLBI data. The second aim was to introduce a selection of the sources into "stable" and "unstable", instead of "defining", "candidates", and "others". Time series used for analysis are listed in table 1.

We used a method of approximation of time series of coordinates by a polynomial model. A linear model with respect to $\beta_{i}(i=0,1,2,3)$ regression polynomial coefficients is

$$
y(t)=\beta_{0}+\beta_{1} t+\beta_{2} t^{2}+\beta_{3} t^{3}+\varepsilon(t),
$$

where $t$ is time, $y(t)$ are corrections $(\Delta \alpha \cos \delta, \Delta \delta)$ to the ICRF coordinates (right ascension or declination) of a source, and $\varepsilon$ is a stochastic value residual. 
Table 1. The list of the time series of the sources' coordinates and codes

\begin{tabular}{lllc}
\hline Name of series & Code & Number of session & Observation interval \\
\hline aus002a & OCCAM 6.2 & 3554 & $1979-04.2007$ \\
bkg00g & CALC/SOLVE 10.0 & 3466 & $1984-07.2007$ \\
dgf000g & OCCAM 6.1 & 2981 & $1984-08.2007$ \\
gsf001a & CALC/SOLVE 10.0 & 4389 & $1979-11.2007$ \\
iaa000b & QUASAR & 4202 & $1979-05.2007$ \\
mao00a & Steelbreeze & 3548 & $1980-05.2007$ \\
opa002a & CALC/SOLVE 10.0 & 3750 & $1984-12.2007$ \\
sai000b & ARIADNA & 3209 & $1984-12.2007$ \\
usn000g & CALC/SOLVE 10.0 & 4170 & $1979-05.2007$ \\
\hline
\end{tabular}

The coefficients of the polynomials were found out by regression analysis. The power of the polynomial was determined by a $R^{2}$ statistic, where

$$
R^{2}=\frac{\sum\left(\hat{y}_{j}-\bar{y}\right)^{2}}{\sum\left(y_{j}-\bar{y}\right)^{2}}=1-\frac{\sum\left(y_{j}-\hat{y}_{j}\right)^{2}}{\sum\left(y_{j}-\bar{y}\right)^{2}} .
$$

Here $y_{j}$ is the correction of right ascension or declination at the moment $t=t_{j}, j=$ $1,2, \ldots, N$, and $\hat{y}_{j}$ is the estimation of polynomial function at $t_{j}$, and $\bar{y}$ is the average value of the series over whole span interval. The value $R$ depends on the correlation between $y$ and $\hat{y}$ (Draper \& Smith, 1998). Obviously, if the polynomial model is correct, that is values of $\hat{y}_{j}$ are equal to $y_{j}$, the coefficient $R=1$. Actually, $\hat{y}_{j} \neq y_{j}$ and $R<1$, but the maximal value of $R$ corresponds to the best fitting model.

As an example, one can see from Fig.1 and Fig.2 that the polynomial model of coordinate variations of the ICRF source $1404+286$ has power larger then 3 . It is well known that when one increases the number of parameters in the fitting function the residual decreases, but the predicted confidence intervals increase enormously. The choice of the model with a small residual and large prediction error, or with a precise prediction and a large residual is determined by the general problem under consideration. At the same time one has to estimate the significance level of regression, i.e. is an increasing $R$ significant or not.
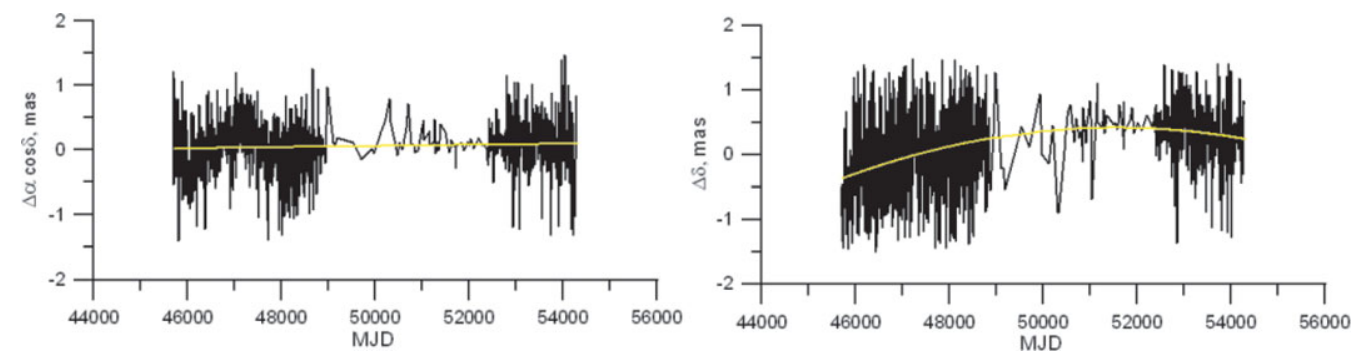

Figure 1. Right ascension variation (left curve) and declination (right curve) of the ICRF source $1404+286$ as function of time. The best fit of $\alpha$ is linear model $\left(\beta_{1}=3 \pm 1 \mu\right.$ as/year $)$, while the best fit of $\delta$ is quadratic polynomial $\left(\beta_{2}=(-3 \pm 2) \times 10^{-4} \mu\right.$ as $/$ year $\left.^{2}\right)$.

We have to estimate the stability of the radio source positions. The stability of the celestial reference frame is determined by sources with negligible apparent velocities. It means that the coefficients $\beta_{i}, i=1,2,3$ in (1.1) have to be close to zero. But analysis shows that many of the ICRF sources have apparent motion, i.e. coefficients $\beta_{i}$ are significantly larger than the errors. Therefore, this fact requests physical explanation. 

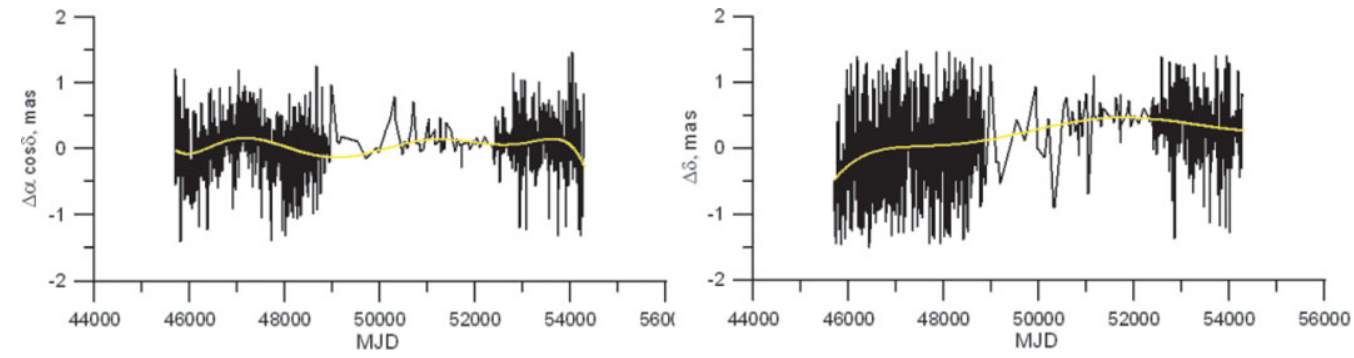

Figure 2. Right ascension variation (left curve) and declination (right curve) of the ICRF source $1404+286$ as function of time. Functions $\alpha$ and $\delta$ were fitted by polynomials of 7 -th power.

To choose the stable ICRF sources we propose the following method of source selection. First of all we consider kinematical characteristics of sources. We can predict the value of $y_{N+1}$ and its confidence intervals outside the observing data span when we have a well-fitted model of $y(t)$.

We call stable sources that show small apparent motion, i.e. the confidence intervals of the predicted corrections to right ascension or declination include a zero value. Otherwise, if the model shows a significant difference of correction to $\alpha$ or $\delta$ from zero, we can call this source unstable (at a corresponding confidence level).

The analysis of data (table 1) shows that many of the ICRF sources reveal significant linear motion, their confidence intervals increase rapidly, and do not include zero. Therefore, we must consider them as unstable in according to the above-mentioned criteria. Actually, one can subtract a well predicted linear trend, and then the confidence intervals include zero and this source can be considered as "stable".

\section{Blandford-Rees model of extragalactic radio sources}

Let us consider a physical mechanism which can explain this apparent motion (Zharov et al., 2009), (Sazhin et al., 2009).

To explain this phenomena we choose the Blandford-Rees (BR) model (Blandford \& Königl, 1979), (Begelman, Blandford \& Rees, 1984). The main idea of this hypothesis is that the quasars and AGN-objects (most of the ICRF sources are quasar or AGN's) represent the system of a massive black hole and jets (see Fig. 3). The optical radiation is formed in black hole's accretion disk while the radio emission is formed into the jet, at some distance from the optical source. Below we will call the radio source a "jet-core" instead of the optical core which coincides with position of massive black hole.

In papers of Zacharias et al. (1999), Assafin et al. (2003) the catalogue of optical positions of 172 ICRF sources was composed. In the first paper a significant spacial difference between optical and radio quasar components was already pointed out. From our point of view this difference indicates that the BR model is appropriate for our purposes. The physical distance between optical and radio components can be estimated for sources with known redshifts and measured angular distances. The mean distance is $300-500 \mathrm{pc}$ (Fig. 4). The uncertainty of distance is connected with uncertainties of the optical positions and the unknown value of the angle between the precession axis and the line of sight. It means that the radio component moves relative to the optical one with the angular velocity $1-10 \mu$ as/year.

We assume that the linear apparent motion can be explained by the precession of the jet while the quadratic apparent motion can be explained by the stochastic process 


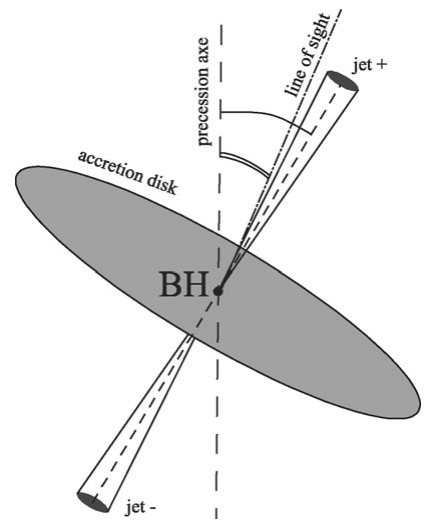

Figure 3. This figure represents the Blandford-Rees model. Central black hole (BH) is surrounded by accretion disk, and two jets from polar regions. The "jet+" is directed to observer. The small black ellipse ending the "+" cone represents a "jet core".

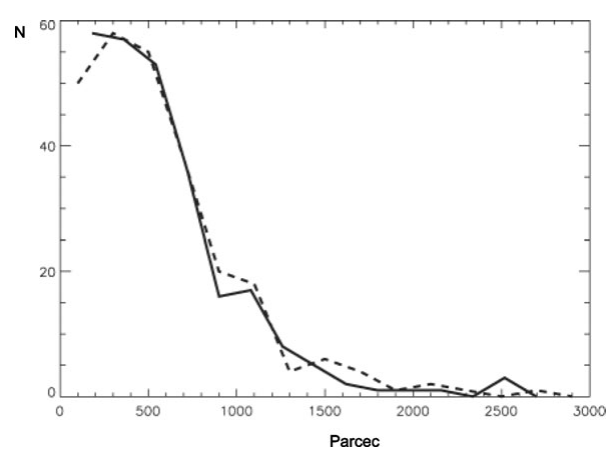

Figure 4. Observed distribution (dashed line) of distances between optical and radio component in quasars according the BR model. The solid line is simulated distribution calculated by the multiplication of two random functions: gamma and sine of a uniformly distributed angle between the precession axis and the line of sight.

of interaction of jet particles with interstellar clouds. The period of jet precession is expected to be $10^{3} \div 10^{6}$ years (Zharov et al., 2009). As long as the precession periods of the jets are significantly larger than the time of observations, the source motions can be treated as linearly stable and predictable with a high accuracy for the time interval of VLBI observations $\sim 30$ years, while the quadratic motion is stochastic and unpredictable.

As a result we restrict our consideration by two models

$$
y(t)=\beta_{0}+\beta_{1} t+\varepsilon(t), \quad y(t)=\beta_{0}+\beta_{2} t^{2}+\varepsilon(t),
$$

and the decision of which model is valid is taken with the following criteria. We calculated the function $R_{1}^{2}$ (1.2) for linear model and $R_{2}^{2}$ (1.2) for a quadratic model. If and only if $R_{2}^{2} / R_{1}^{2}>5$, we accept the quadratic model of apparent motion. Approximately two-thirds of the sources show linear and one-third show quadratic motion.

As long as linear motion is predictable and stationary for a long time interval, one can subtract the linear trend of the data and work with these "residual" data. 


\section{Cosmological criterion for ICRF sources choice.}

All other motions inside the radio source represent a noise component of astrometric observation. These motions occur inside some linear scale. The shorter scale the smaller angular displacement as seen by an observer.

Thereby to decrease astrometric noise and to improve the coordinate system stability we have to choose the most remote sources. It is correct in the Euclidean space: the more remote a source the less the angular scale of its apparent motion. In the Friedman model of expanding Universe it is not correct. Extragalactic objects have to be considered in expanding space-time, and in framework of the Standard Cosmological Model.

According to this model the apparent angular size of the source has a minimum for an redshift $z=1.63$. An object located at this distance with a physical size of about $1 \mathrm{pc}$ has an angular size equals to $\theta=116 \mu a s$. This is a minimal angular size of an object, and it will increase for $z<1.63$ and for $z>1.63$. It was shown that the interval of redshift $0.8 \leqslant z \leqslant 3.0$ is the most favorable in terms that the physical shift inside such sources corresponds to minimal apparent angular shift of a "jet core". Details of this calculation can be found in (Sazhin et al., 2009).

After the selection of sources as "unstable" and "stable" according to the "kinematical" and "cosmological" criteria we obtained the final list of 137 sources (see Table 1 in (Sazhin et al., 2009)).

\section{The ICRF system instability.}

As was pointed out that the main purpose of the selection of "stable" sources is the stability of the celestial reference frame which is connected with the predictability of the source motion.

The variation of the ICRF source coordinates leads to a small rotation of reference system. To estimate the stability of the system three small angles $\theta_{1}, \theta_{2}, \theta_{3}$, which describe a small rotation were calculated:

$$
\mathbf{s}(t)=\left(\begin{array}{ccc}
1 & -\theta_{3} & \theta_{2} \\
\theta_{3} & 1 & -\theta_{1} \\
-\theta_{2} & \theta_{1} & 1
\end{array}\right) \mathbf{s}\left(t_{0}\right)
$$

where $\mathbf{s}(t), \mathbf{s}\left(t_{0}\right)$ are unity vectors of a source at moments $t$ and $t_{0}=J 2000.0$.

In the Fig. 5 the angles $\theta_{1}, \theta_{2}, \theta_{3}$, calculated for different subsets of the ICRF sources ("defining", "stable" and for "cosmological") are shown.
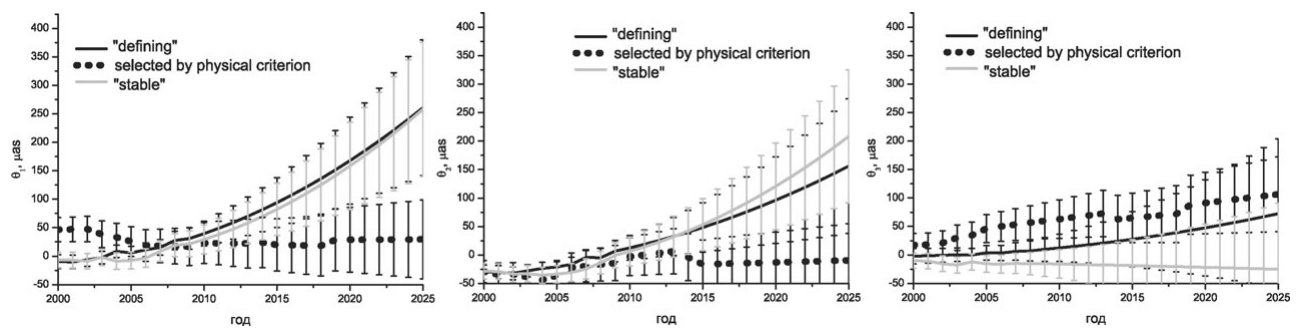

Figure 5. The rotation angles $\theta_{1}(\mathrm{a}), \theta_{2}(\mathrm{~b}), \theta_{3}(\mathrm{c})$ calculated for different subsets of the ICRF sources: defining (black), the Feissel's stable (gray) and our list based on physical criteria (dotted).

Obviously the subset formed on bases of the cosmological criteria makes the stability of the ICRF better. 


\section{Conclusions}

The physical basis of "cosmological" and "kinematical" criteria is based on the assumption that apparent motion of quasars is connected with real motion inside quasars. Therefore apparent angular motion corresponds to a real physical shift of a "jet core" inside a radio source. The interval of redshift $0.8 \leqslant z \leqslant 3.0$ is the most favorable in terms that physical shift inside such sources corresponds to a minimal apparent angular shift of a "jet core". The method of "cosmological" selection improves stability of the ICRF over next decade.

\section{Acknowledgements}

Authors thank Dr. D. Gabuzda, Prof. K. Postnov, Prof. B. Somov, and Dr. O. Titov for fruitful discussion. This work has been supported by Russian Foundation for Basic Research grants 07-02-01034 and 08-02-00971, grant of the President of RF MK-2503.2008.2 (O.S.).

\section{References}

Assafin, M., Zacharias, M., Rafferty, T. J. et al., 2003, AJ, 125, 2728

Begelman, M. C., Blandford, R. D., \& Rees, M. J., 1984, Rev. Mod. Phys, 56, 255

Blandford, R. D. \& Königl, A., 1979, AJ, 232, 34

Draper, N. R. \& Smith, H. Applied Regression Analysis. 3-rd edition. John Wiley \& Sons, Inc. 1998.

Ma, C., Arias, E. F., Eubanks, T. M., et al., AJ, 116, 516, 1998.

Sazhin, M. V., Sementsov, V. N., Zharov, V. E., Kuimov, K. V., Ashimbaeva, N. T., \& Sazhina, O. S., 2009, arXiv:0904.2146v1

Zacharias, N., Zacharias, M. I., Hall, D. M. et al., 1999, AJ, 118, 2511

Zharov, V. E., Sazhin, M. V., Sementsov, V. N., Kuimov, K. V., \& Sazhina, O. S., AZh, 86, 527, 2009 (in russian); Astron. Rep., 53, 579, 2009. 